\title{
Research Paper \\ The Awareness and Attitude of General Dentists on Dental Implants Follow-up
}

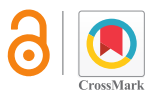

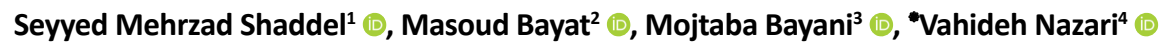

1. Department of Dental Prostheses, School of Dentistry, Kashan University of Medical Sciences, Kashan, Iran.

2. Student Research Center, Arak University of Medical Sciences, Arak, Iran.

3. Department of Periodontology, School of Dentistry, Arak University of Medical Sciences, Arak, Iran.

4. Department of Dental Prostheses, School of Dentistry, Arak University of Medical Sciences, Arak, Iran.

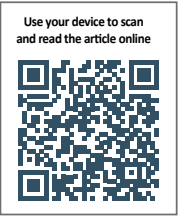

Citation: Shaddel SM, Bayat M, Bayani M, Nazari V. [The Awareness and Attitude of General Dentists on Dental Implants Follow-up (Persian)]. Journal of Arak University of Medical Sciences (JAMS). 2021; 24(2):268-277. https://doi.org/10.32598/ JAMS.24.2.6209.1

doi https://doi.org/10.32598/JAMS.24.2.6209.1

Keywords:

Dental implant, Follow-Up, Dentist, Awareness, Attitude

\section{ABSTRACT}

Article Info:

Received: 07 Jun 2020

Accepted: 06 Jan 2021

Available Online: 01 Jun 2021

\section{Extended Abstract}

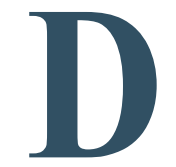

\section{Introduction}

ental implants are among the best treatment methods to restore function and beauty. The long-term success of implants depends on the follow-up sessions [1]. In the first year after treatment, regular visits should be at intervals of 3 months, then determined according to the individual's needs [2]. Dental implants are prone to mechanical problems and plaque-related inflammatory changes over time [3].

\section{* Corresponding Author:}

Vahideh Nazari, PhD.

Address: Department of Dental Prostheses, School of Dentistry, Arak University of Medical Sciences, Arak, Iran.

Tel: +98 (918) 7595078

E-mail: nazari.vahideh@yahoo.com 
Various methods have been used to assess the health of the implant during follow-up [2, 4]. Soft tissue and implant prosthesis should be examined. Intraoral radiography should be also provided [5-7].

As the demand for patients for implants has dramatically increased, dentists must increase their knowledge and awareness. Therefore, this study aimed to investigate the knowledge and attitude of general dentists about follow-up implants to adjust the appropriate program and educational content.

\section{Materials and Methods}

The present study analytical and cross-sectional study was conducted in Arak City, Iran, in 2019. The questions were organized into 3 sections, as follows: demographic, awareness, and attitude. To design the questions, the opinions of experts in the fields of the prosthesis, radiology, and periodontology, as well as using reference books were used $[8,9]$. The first part of the questionnaire concerned demographic characteristics.

The second part was related to the dentist's knowledge of follow-up of implant treatment. The third section included questions about the dentist's attitude toward implant followup. To determine validity and reliability, 40 questionnaires were completed. The validity and reliability of the questionnaire were confirmed by calculating Cronbach's alpha coefficient of $90 \%$. The validity and reliability of this questionnaire were measured to be $75 \%$ and $86 \%$, respectively.

In the questions section, the correct answers were given a score of 1 . The maximum score in this section equaled 19 . In the attitude questions section, the low answer was given 1 score, the average answer was given 2 scores and the high answer was given 3 scores; thus, the maximum score was equal to 21. Statistical analysis was performed using the Chi-squared test and Independent Samples t-test.

\section{Results}

Among the 98 active dentists in Arak who participated in the study; 41 were female and 57 were male. In total, $26.8 \%$ had 1-3 years of work experience, $13.2 \%$ had 3-5 years work experience, $42.8 \%$ had 5-10 years work experience, and $17.4 \%$ had 10 years or more work experience. From the maximum score of 19 in the knowledge section, the mean obtained scores were measured as 10.8; in the attitude section, of the maximum score of 21 , the mean scores equaled 12.8. The number of years of work experience did not significantly affect the examined dentists' knowledge and attitude about the follow-up of implant treatment $(\mathrm{P}>0.05)$.

There was a statistically significant difference in the mean knowledge and attitude values of dentists who underwent implant treatment and those who did not (Table 1). Dentists who performed implant treatment were significantly more aware of implant treatment follow-up in all areas of knowledge (dental prostheses, radiology, \& periodontology). Moreover, in the attitude questions section, dentists who performed implant treatment received 14.5 points; dentists who did not perform implant treatment received 11.1 points. There was no significant gender-wise difference in the mean scores of knowledge and attitude $(\mathrm{P}>0.05)$. Dentists achieved the highest knowledge score in periodontology; they obtained the lowest knowledge in prosthetics. However, this difference was not statistically significant $(\mathrm{P}>0.05)$.

Table 1. The awareness and attitude levels of dentists based on implant treatment

\begin{tabular}{ccccc}
\hline Variabels & Implant Treatment & No. & Mean & P \\
\hline Periodontology & Yes & 51 & 8.0 & 0.000 \\
& No & 47 & 6.4 & 0.026 \\
Radiology & Yes & 51 & 7.8 & 0.012 \\
\hline
\end{tabular}


Table 2. The awareness level of the explored dentists concerning radiology, prosthetics, and periodontology based on implant treatment

\begin{tabular}{ccccc}
\hline Variabels & Implant Treatment & No. & Mean & P \\
\hline \multirow{2}{*}{ Awareness } & Yes & 51 & 12.1 & 0.0001 \\
& No & 47 & 9.5 & 0.0001 \\
\hline
\end{tabular}

Journal of

Arak University of Medical Sciences

\section{Discussion and Conclusion}

The present study data indicated that the level of knowledge and attitude of general dentists about dental implant follow-ups was low. In the study of haghighat et al., the average score of knowledge obtained was far from the full score [10]. This finding indicated the inadequacy of implant education in the entire dental education system. In a study by Jayachandran et al., 77\% of general dentists were trained in only theoretical aspect of implant [11].

The results revealed that dentists who had performed implant treatment had awareness and attitude toward implants follow-up higher than those of the dentists who did not perform dental implant treatment. According to Gibson's study, general dentists believed that implant treatment should be performed by a specialist dentist [12].

Levin et al. found that $80 \%$ of general dentists feel the need for implant training courses [13]. Nagpal stated that general dentists presented the most negative attitude concerning implant treatments [14]. According to the obtained results, there was a slight difference in knowledge and attitude between dentists who had different years of work, i.e., not statistically significant. Dentists with 3-5 years of work experience had the best knowledge and the most positive attitude towards implant treatment, i.e., consistent with Nagpal's study [14].

The study suggested that gender had no significant effect on dentists' knowledge and attitude. According to Trovic, age, gender, and place of work of the dentist had no effect on their decision regarding the edentulous patient [13]. The current research results indicated the lack of knowledge and attitude of general dentists about the follow-up of dental implants. It is necessary to present further implant training classes for students and retraining classes for general dentists.

\section{Ethical Considerations}

\section{Compliance with ethical guidelines}

This article was approved by the Ethics Committee of Arak University of Medical Sciences (Code: IR.ARAKMU. REC.1397.283).

\section{Funding}

This study was supported by the Vice Chancellor for Research of Arak University of Medical Sciences.

\section{Authors' contributions}

All authors met the standard writing criteria based on the recommendations of the International Committee of Medical Journal Publishers (ICMJE).

\section{Conflicts of interest}

The authors declared no conflict of interest.

\section{Acknowledgements}

The authors thank the Arak University of Medical Sciences for its technical support in conducting this research study. 


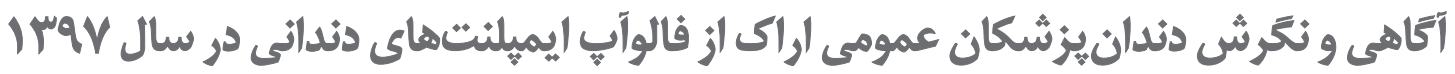

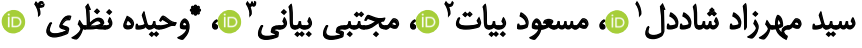

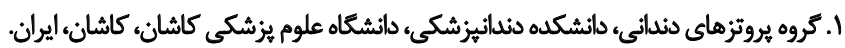

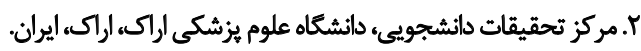

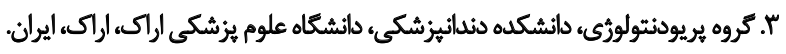

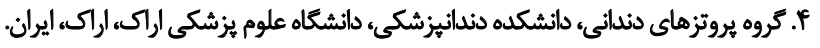

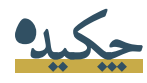

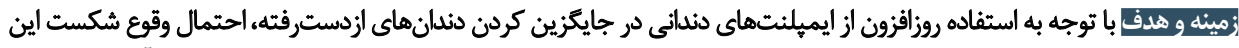

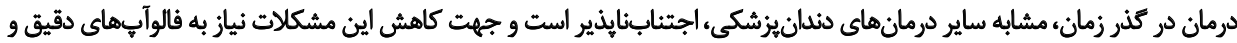

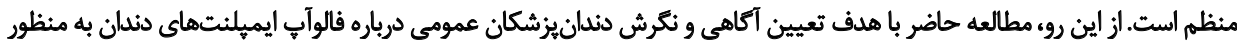

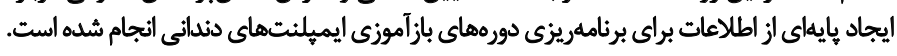

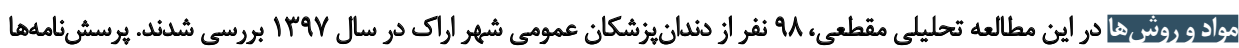

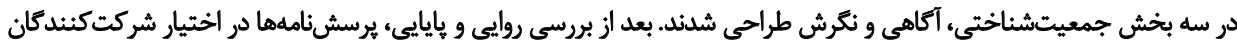

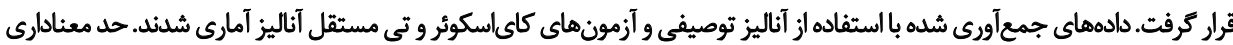

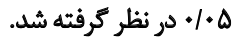

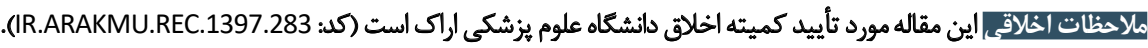

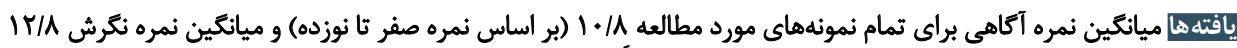

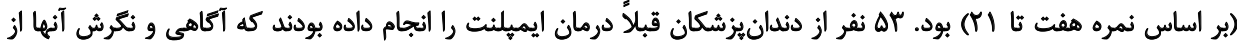

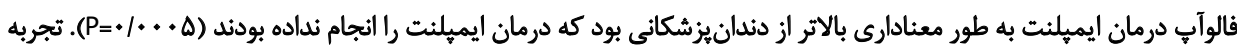

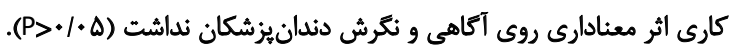

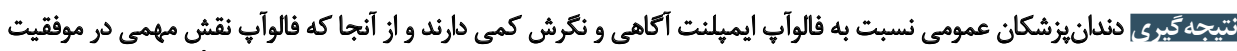

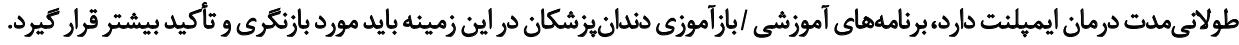

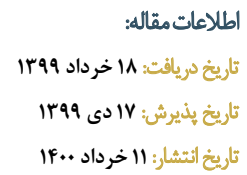

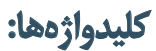

ايميلنت دندانى، فالوآي،

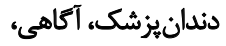
نئرش ندان
بايد در فواصل سه ماه باشد و سيس متناسب با نيازهاى فردى

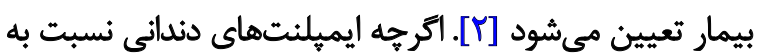

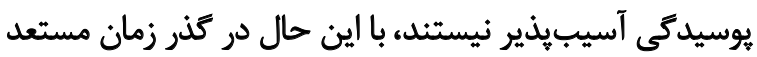

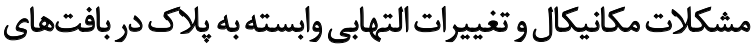

اطراف ايميلنت هستند [ب]ت

جهت ارزيابي سلامت ايميلنت و بافتهاي اطرافي

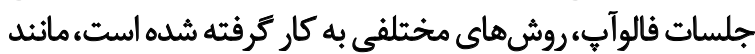

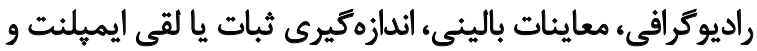

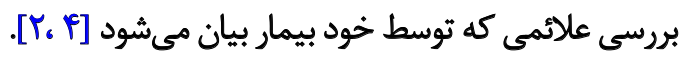

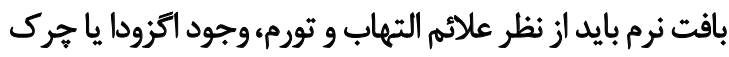

doläo

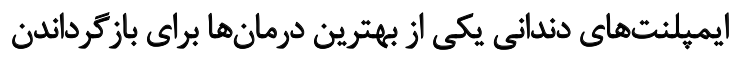

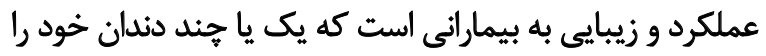

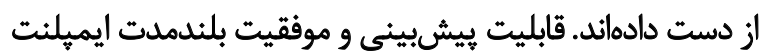

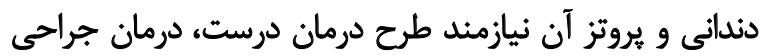

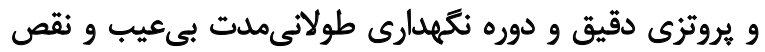

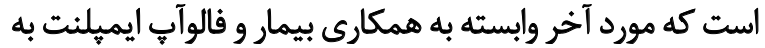

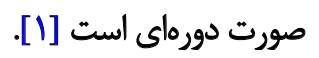

در سال اول يس از درمان، مراجعات منظم به دندانيزشك 
با محاسبه ضريب آلفاى كرونباخ · م درصد و نظرات مشاور آمار

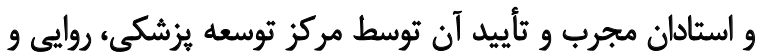

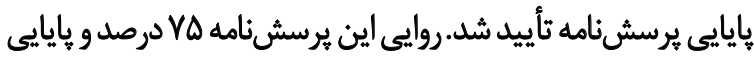

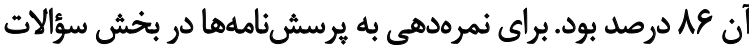

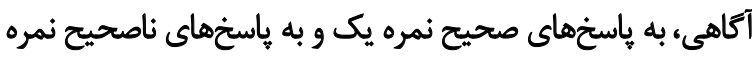

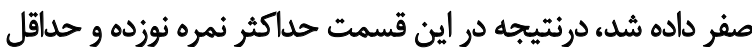

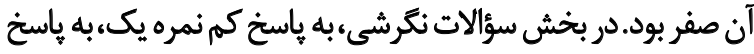

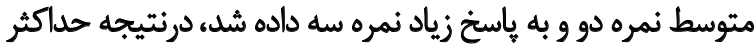

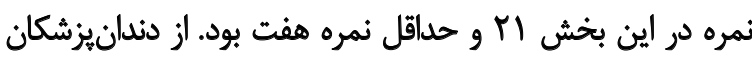

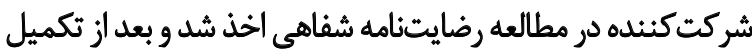

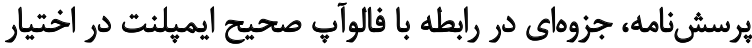

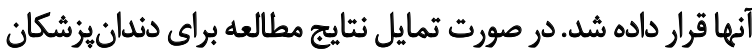

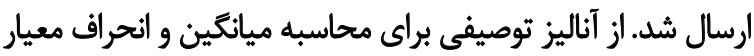

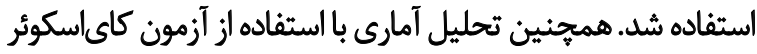

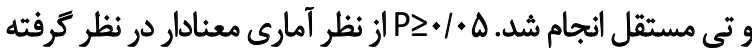

Larọl

از ميان هو دندانيزشك فعال در شهر اراك كه در تحقيق

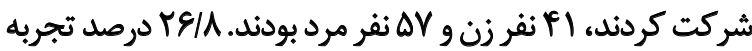

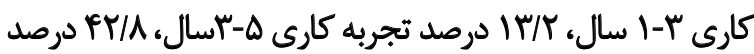

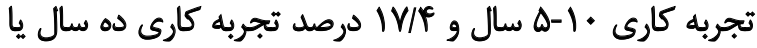

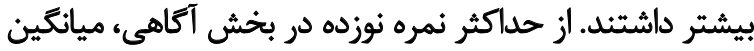

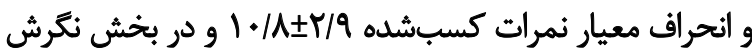

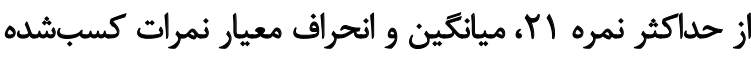

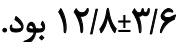

بيشترين نمره آكاهى و نكُش مربوط به كروه با تجربه كارى

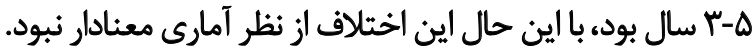

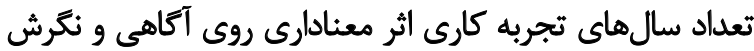

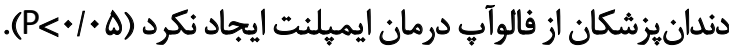

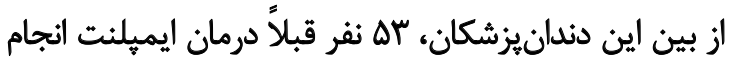

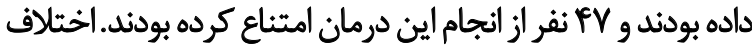

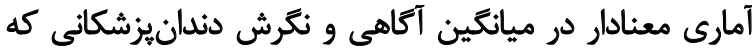

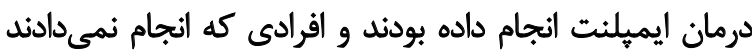

وجود داشت (جدول شماره ()).

دندانيزشكانى كه درمان ايميلنت انجام مي دادند، به طور

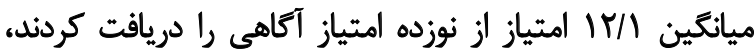

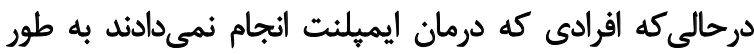

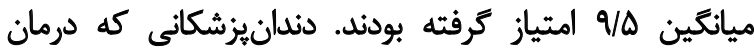

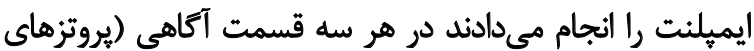

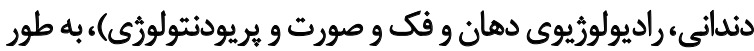

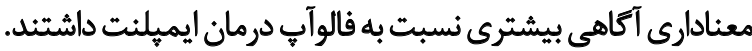

و عمق يروب معاينه شود. راديوكرافي داخل دهانى يرياييكال

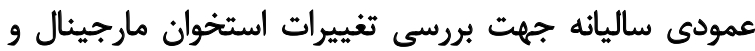

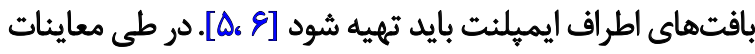

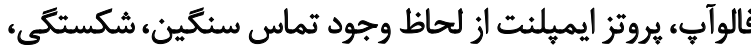

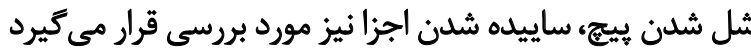

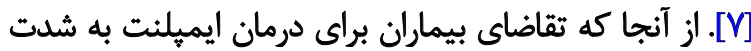

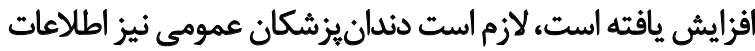

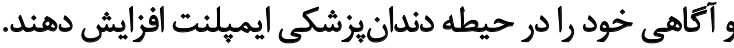

بديهي است دندانيزشكانى كه درمان ايميلنت را انجام

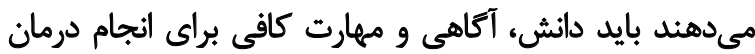

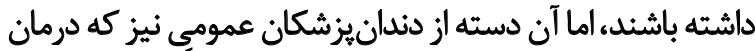

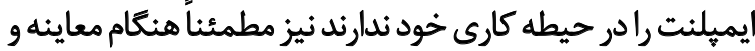

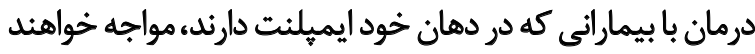

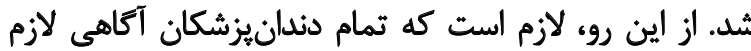

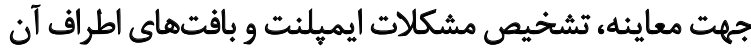

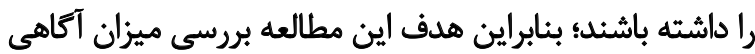

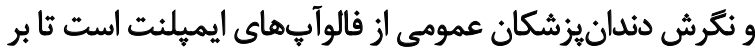

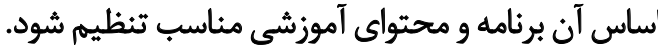

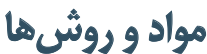

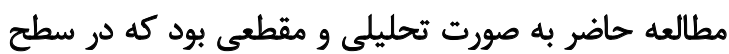

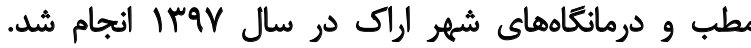

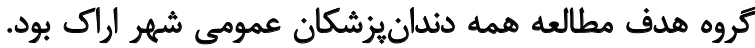

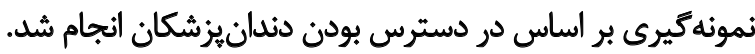
11 9ندانيزشك عمومى وارد مطالعه شدند.

بر اساس اهداف اختصاصى يُروهش، سؤالات در سه بخش

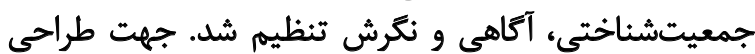

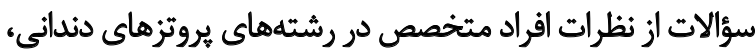

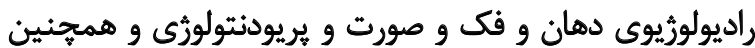

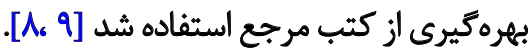

فخش اول يرسشنامه مربوط به مشخصات جمعيتشناختى

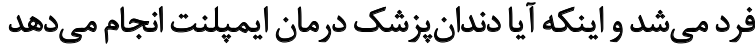

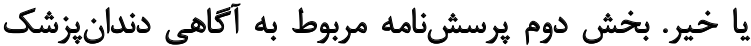

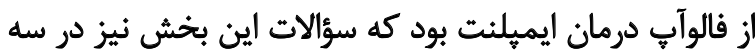

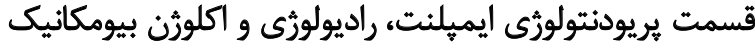

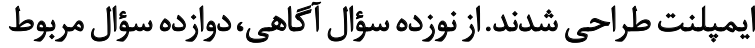

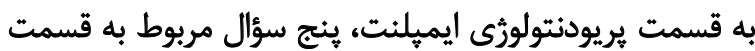

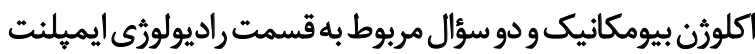

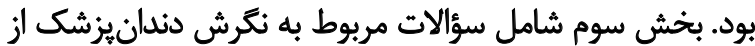

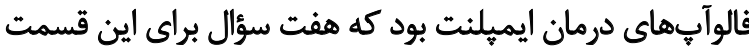

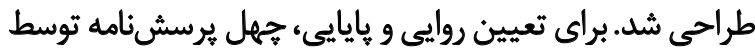

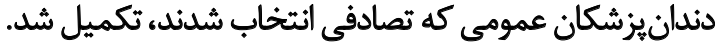


جدول أ. آكاهى و نتكرش دندانيزشكان بر حسب انجام درمان ايميلنت

\begin{tabular}{|c|c|c|c|c|}
\hline $\mathbf{P}$ & مياتكين & تعداد & درمان إيميلنث & مثغير \\
\hline \multirow{3}{*}{$.10+\infty 1$} & $\mid r / 1$ & $\Delta 1$ & بله & \multirow{3}{*}{ أكاهي } \\
\hline & & & & \\
\hline & V/D & p & خير & \\
\hline \multirow{2}{*}{$.1+\ldots 1$} & $\mid F / \Delta$ & Q1 & بله & \multirow{2}{*}{ نكاش } \\
\hline & $11 / 1$ & pr & خير & \\
\hline
\end{tabular}

اين امر حاكي از ناكافي بودن آموزش مربوط به ايميلنت در كل

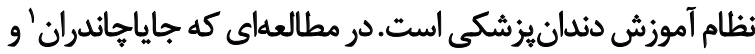

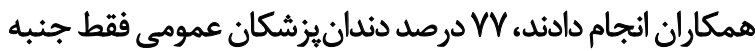

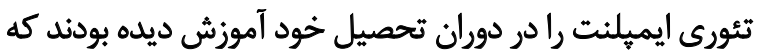

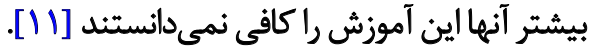

نتايج نشان داد دندانيزشكاني كه درمان ايميلنت را انجام

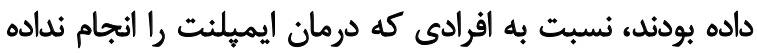

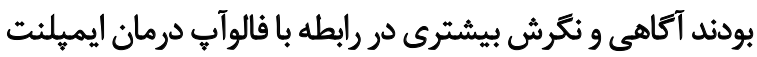

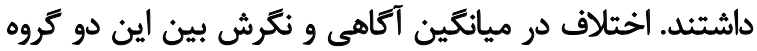

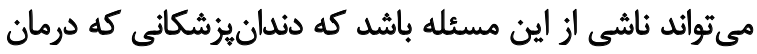

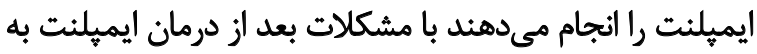

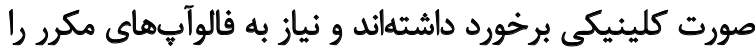
احساس كردهاند.

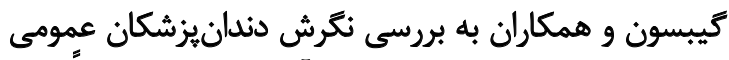

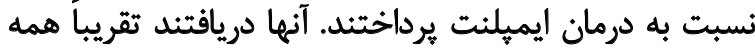

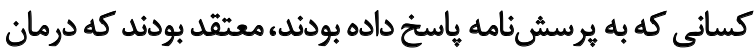

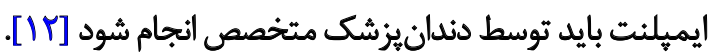
مطالعه حاضر نشان داد با وجود آكاهي و نئرش كم إنم

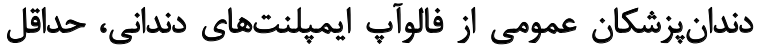

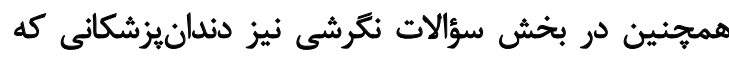

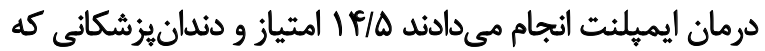

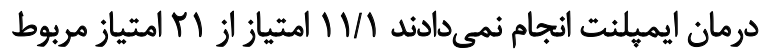

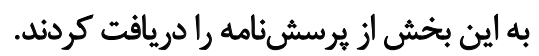

اختلاف آمارى معنادار در ميانكين آكاهي و نكَش بين خانمها

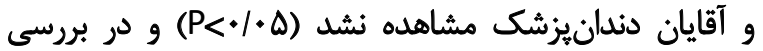

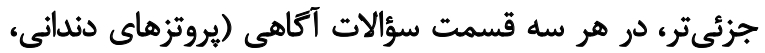

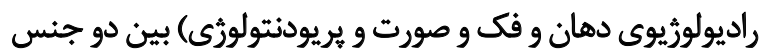

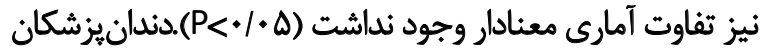

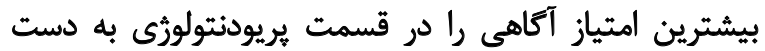

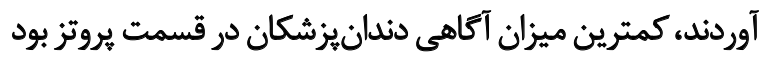

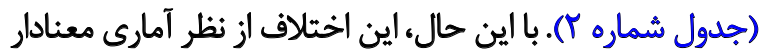

نبود (Q)

\&)

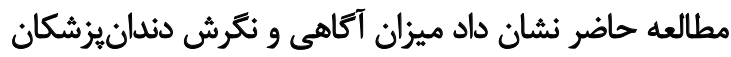

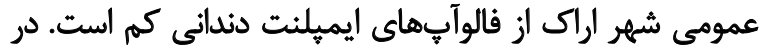

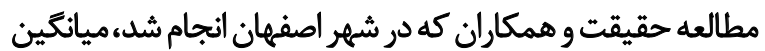

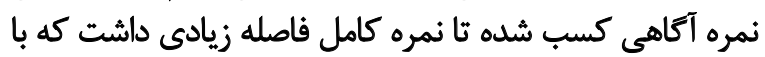

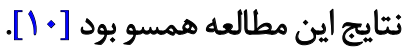

جدول r. آثاهى دندانيزشكان در قسمتهاى يريودنتولوزىى، راديولوزى، يروتز بر حسب انجام درمان ايميلنت

\begin{tabular}{|c|c|c|c|c|}
\hline $\mathbf{P}$ & هتوسط & تعداد & ورمان ايميلنت & متغير \\
\hline \multirow{2}{*}{$10+0$} & $N$. & a) & بله & \multirow{2}{*}{ بريودنتولوزى } \\
\hline & $g / f$ & PV & خير & \\
\hline \multirow{2}{*}{ /.rg } & $\mathrm{V} / \mathrm{A}$ & Q1 & بله & \multirow{2}{*}{ راديولوزى } \\
\hline & 91. & PV & خير & \\
\hline \multirow{2}{*}{.$/ \cdot 1 r$} & $V / q$ & DI & بله & \multirow{2}{*}{ بروتز } \\
\hline & $\Delta / \lambda$ & PV & خير & \\
\hline
\end{tabular}


دندانيزشك، در رابطه با بيمار بي دندان ندارد [ [ [1]. علت عدم ثفاوت

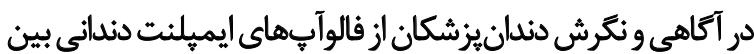

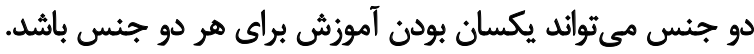

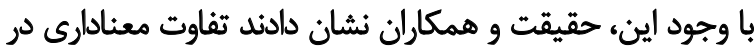
ميانكين آكاهى بين خانمها وآقايان وجود دارد [ • إن.

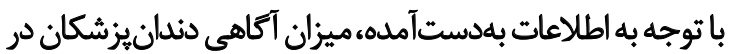

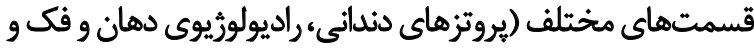

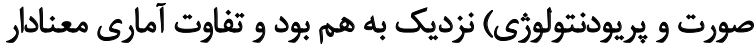
در اين سه قسمت مشاهده نشد.

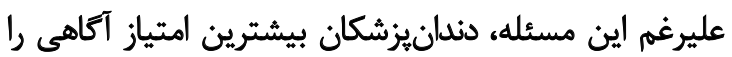

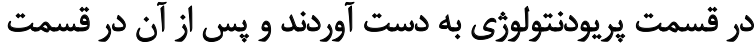

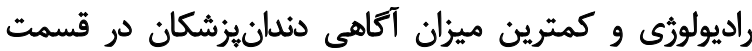

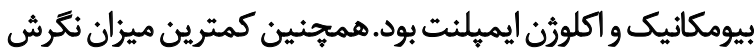

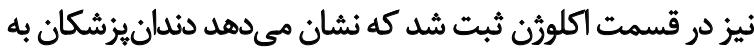

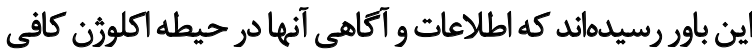

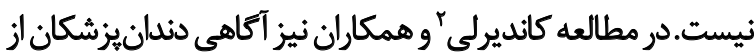

اكلورن كافى نبود [1ه].

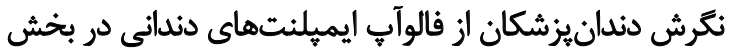

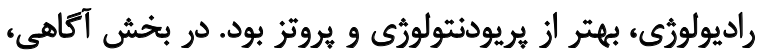

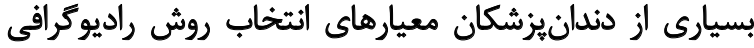

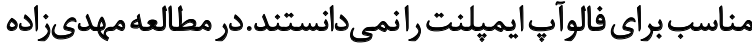

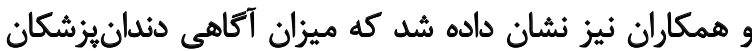
عمومى شهر اصفهان در زمينه تجويز صحيح راديوكرافى كمتر ازئ اندان

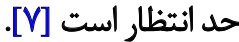

علث اين اختلاف بين آكاهى از فالوآب و نكرش از فالوآي

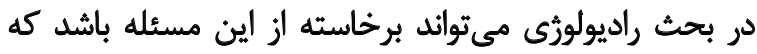

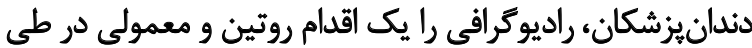

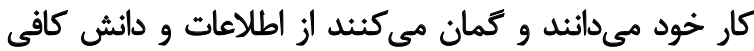

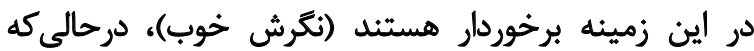

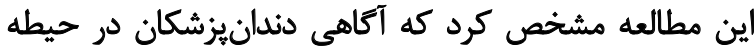

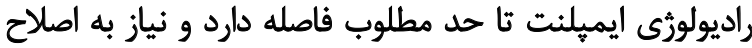

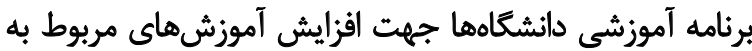

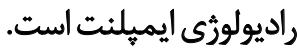

\section{نتيجليكّى}

نتايج اين مطالعه حاكى از كم بودن آكاهى و نكّرش

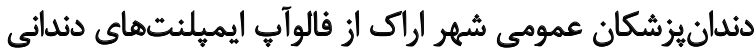

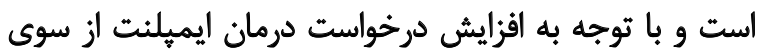

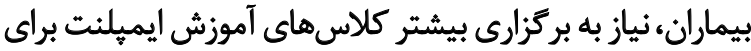

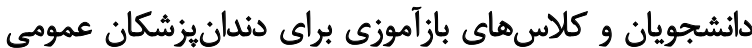

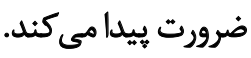

نيمى از دندان يزشكان عمومى درمان ايميلنت را انجام مي دهند.

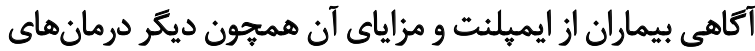

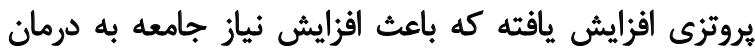

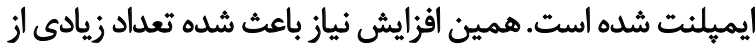

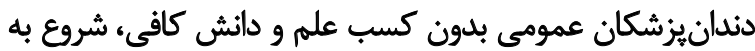

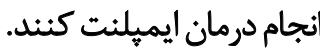

صرفه اقتصادى و سهولت انجام درمان ايميلنت نسبت به بعضى دئي

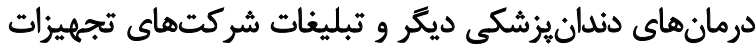

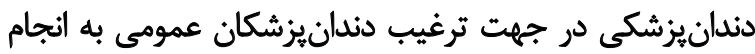

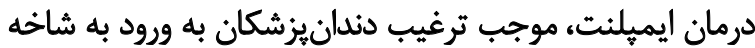

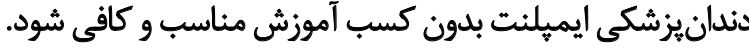

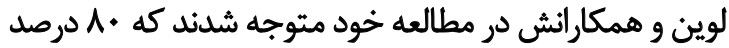

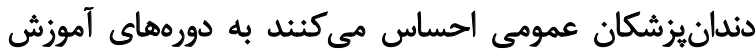

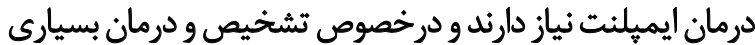

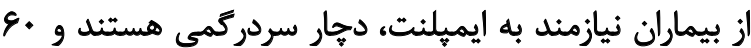

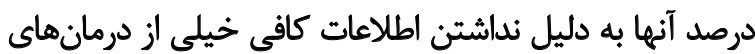

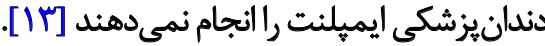
نتايج مطالعه ناكيال و همكارانش حاكى از آن بود كهي

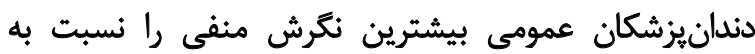

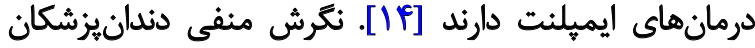

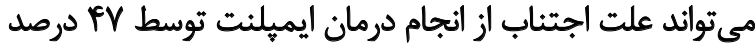
دندانيزشكان عمومى در مطالعه حاضر باشند.

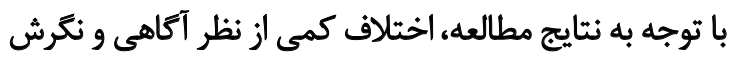

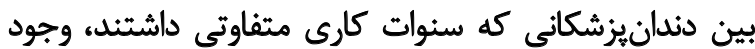

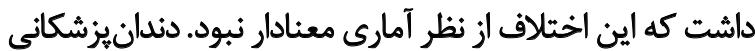

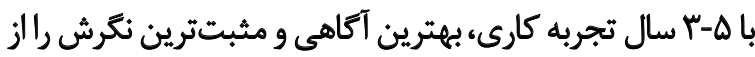

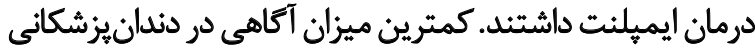
با تجربه كارى بيش از ده سال مشاهده شد.

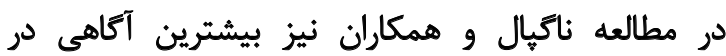

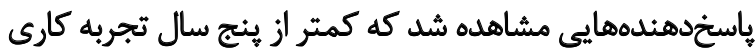

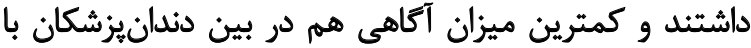

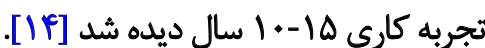

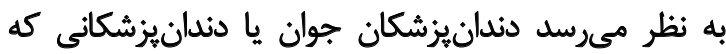

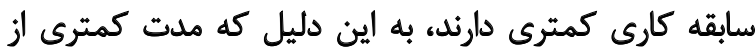

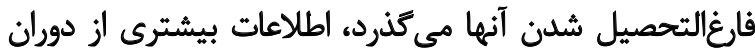
تحصيلات دانشكاهى خود به ياد انها دارند.

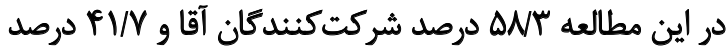

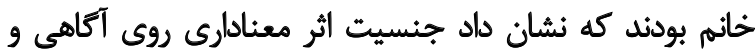

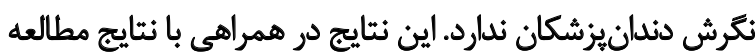

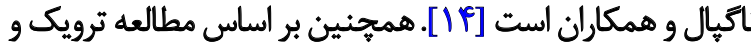

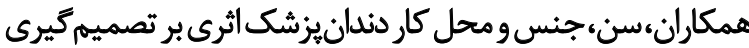




\section{مالاحظات اخلاقى}

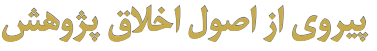

اين مقاله مورد تأييد كميته اخلاق دانشكاه علوم يزشكى اراك با شناسه اخلاق

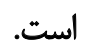

$$
\text { مانم }
$$

معاونت يزؤودشى دانشكاه علوم يزشكى اراك از اين مقاله

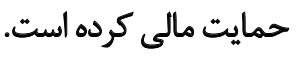

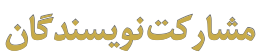

تمام نويسندكان معيارهاى استاندارد نويسندگى بر براس اساس

بيشنهادات كميثه بينالمللى ناشران مجلات يزشكيى (ICMJE) را دارا بودند.

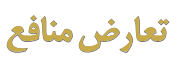

بنابر اظهار نويسندكان اين مقاله تعارض منافع ندارد.

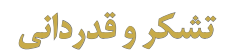

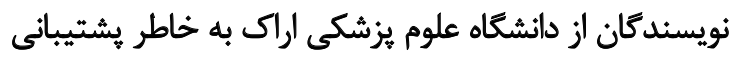
فنى در اجراى اين مطالعه تحقيقاتى قدردانى مى كنيند. 


\section{References}

[1] Rosen PS, Sahlin H, Seemann R, Rosen AS. A 1-7 year retrospective follow-up on consecutively placed 7-mm-long dental implants with an electrowetted surface. Int J Implant Dent. 2018; 4(1):24. [DOI:10.1186/ s40729-018-0136-4][PMID][PMCID]

[2] Kanathila H, Pangi A, Benakatti V, Patil S. Maintenance of dental implants: A way to long term success: A review. Int J Appl Dent Sci. 2018; 4(2):104-7. https://www.researchgate.net/publication/327594119_ Maintenance_of_dental_implants_A_way_to_long_term_successA_review

[3] De Angelis F, Papi P, Mencio F, Rosella D, Di Carlo S, Pompa G. Implant survival and success rates in patients with risk factors: Results from a long-term retrospective study with a 10 to 18 years follow-up. Eur Rev Med Pharmacol Sci. 2017; 21(3):433-7. [PMID]

[4] Gulati M, Govila V, Anand V, Anand B. Implant maintenance: A clinical update. Int Sch Res Notices. 2014; 2014:908534. [DOI:10.1155/2014/908534] [PMID] [PMCID]

[5] Etter TH, Håkanson I, Lang NP, Trejo PM, Caffesse RG. Healing after standardized clinical probing of the perlimplant soft tissue seal: A histomorphometric study in dogs. Clin Oral Implants Res. 2002; 13(6):571-80. [DOI:10.1034/j.1600-0501.2002.130601.x] [PMID]

[6] Newman MG, Flemmig TF. Periodontal considerations of implants and implant associated microbiota. Int J Oral Implantol. 1988; 5(1):65-70. [PMID]

[7] Mahdizadeh M, Fazaelipour M, Namdari A. [Evaluation of dentistsi ${ }^{-}$ awareness of how to prescribe correct radiographs in Isfahan in 20102011 (Persian)]. Dent Res J (Isfahan). 2012; 7(5):637-42. http://jids. journalonweb.ir/index.php/jids/article/view/443

[8] Newman MG, Takei HH, Klokkevold PR, Carranza FA. Carranza's clinical periodontology. Philadelphia: Saunders Elsevier; 2015. https://books. google.com/books?id=LFTjAQAACAAJ\&dq

[9] Misch CE. Dental implant prosthetics. Maryland Heights: Elsevier Mosby; 2014 . https://books.google.com/books?id=1iBtngEACAAJ\&dq

[10] Haghighat A, Bonakdarchian M, Ghafari SM. [Evaluation of Isfahan dental practitioner's awareness about dental implants (Persian)]. Dent Res J (Isfahan). 2011; 6(4):493-9. https://jids.journalonweb.ir/index. php/jids/article/view/249

[11] Jayachandran S, Bhandal BS, Hill KB, Walmsley AD. Maintaining dental implants--do general dental practitioners have the necessary knowledge? $\mathrm{Br}$ Dent J. 2015; 219(1):25-8. [DOI:10.1038/sj.bdj.2015.528] [PMID]

[12] Gibson RL, Barclay CW. Dental implantology education: A survey of opinion and experience of 106 general dental practitioners. Br Dent J. 2006; 201(6):367-70. [DOI:10.1038/sj.bdj.4814043] [PMID]

[13] Pournasrollah A, Negahdari R, Vahid Pakdel SM. Evaluation of the knowledge of general dental practitioners in Tabriz about the implant treatment planning. Adv Biosci Clin Med. 2015; 3(3):24-31. http://www. journals.aiac.org.au/index.php/ABCMED/article/view/1546

[14] Nagpal D, Prakash S, Kalra DD, Singh G. Knowledge, attitude, and practice of dental implants among dental postgraduates and practitioners in Davangere City, Karnataka: A cross-sectional study. Indian J Dent Res. 2018; 29(5):575-82. [DOI:10.4103/ijdr.IJDR_500_15] [PMID]

[15] Candirli C, Korkmaz YT, Celikoglu M, Altintas SH, Coskun U, Memis S. Dentists' knowledge of occlusal splint therapy for bruxism and temporomandibular joint disorders. Niger J Clin Pract. 2016; 19(4):496-501. [DOI:10.4103/1119-3077.183310] [PMID] 
This Page Intentionally Left Blank 